\title{
Measurement of Peripheral Blood Mononuclear Cells Producing IFN-Gamma in Patients With Tuberculosis
}

\author{
Dahir Ramos de Andrade Júnior ${ }^{1}$, Sânia Alves dos Santos ${ }^{2}$ and Dahir Ramos de Andrade ${ }^{2}$ \\ ${ }^{1}$ Department of Internal Medicine, Medical Investigation Laboratory - LIM 54, School of Medicine, University of São Paulo; ${ }^{2}$ Department of Infectious \\ and Parasitary Diseases, Medical Investigation Laboratory - LIM 54, School of Medicine. University of São Paulo; São Paulo, SP, Brazil
}

\begin{abstract}
The type of immune response induced by tuberculosis (Th1 or Th2) and its correlation with the clinical outcome is unclear. We studied 13 patients with active tuberculosis (TBC). The peripheral blood mononuclear cells producing IFN-gamma (PBMC-IG) were measured by enzyme-linked immunospot (ELISPOT) technique. The control group had ten healthy individuals vaccinated against tuberculosis. We collected blood samples of each patient in two moments: a) in the hospital admission without treatment (TBC1); b) after seven to 20 days of treatment (TBC2). The comparison of the spots forming units of PBMC-IG between TBC group and controls showed that there was a significant difference between TBC1 and control group $(p<0.001)$ and between TBC2 and control group $(p<0.005)$, but there was no difference between TBC1 and TBC2 $(p>0.05)$. A positive correlation was found between PBMC-IG and hemoglobin value, as well as between PBMC-IG and weight loss. There was no correlation between PBMC-IG and other variables [age, erythrocyte sedimentation rate (ESR), C-reactive protein (CRP)]. We conclude that tuberculosis activates Th1 immune response due to increase of PBMC producing IFN-gamma. There was no difference between the first sample (TBC1) and the second sample (TBC2) of PBMC-IG. This result can have occurred due to treatment influence, or can indicate that the immune response reachs a plateau. The positive correlation among PBMC-IG and both hemoglobin level and weight loss indicates that may exist a link between patient's clinical status and the immune response intensity.
\end{abstract}

Key-Words: Tuberculosis, Th1 immune response, ELISPOT.

The characterization of $\mathrm{T}$ cell responses by measurement of cytokine secretion by ELISPOT assay has become widely used, mainly in the vaccine production, cancer immunotherapy $[1,2]$, and in the immune response evaluation against infectious agents $[1,3,4]$.

The role of cytokines produced by Th1/Th2 lymphocytes is complex due to the action of TNF-alpha, IL-12, IFN-gamma (pro-inflammatory cytokines) and IL-4, IL-10 and TGF-beta (anti-inflammatory cytokines). Th1 lymphocytes produce proinflammatory cytokines while Th2 lymphocytes produce antiinflammatory cytokines. Normally, the Th1 immune response is correlated with tissue injury. In this way, the clinical form of infectious diseases, as well as the cell death intensity will be determined by the predominant immune response, Th1 or Th2. In patients with tuberculosis (TBC) and PPD skin test positive the predominant immune response may be Th1, although there are few studies in this field.

The cellular immune response is essential for tuberculosis resistance, mainly through cytokines production such as IL12, IL-18, IFN-gamma, IL-2 and TNF-alpha. The CD8+ T cells perform cytotoxic action mainly through major histocompatibility complex (MHC) class I molecules in association with CD1d molecules presenting glycolipids antigens to T lymphocyte cells [5]. Cytotoxic T lymphocyte

Received on 12 December 2007; revised 6 April 2008.

Address for correspondence: Dr. Dahir Ramos de Andrade Júnior, MD. Av. Paes de Barros 701, ap. 101, Mooca, São Paulo, São Paulo, Brazil. Zip code: 03115-020. Phone: 0115511 - 30617029. Fax: 0115511 30617029. Email: dahira@uol.com.br.

Financial support: The principal author received financial support from FAPESP (Fundação de Amparo à Pesquisa do Estado de São Paulo).

The Brazilian Journal of Infectious Diseases 2008;12(2):123-127. (C) 2008 by The Brazilian Journal of Infectious Diseases and Contexto Publishing. All rights reserved. cells (CTLs) may have a protective role in TBC disease by several mechanisms: 1) Producing potent antibacterial cytokines such as IFN-gamma and TNF-alpha; 2) Presenting M. tuberculosis antigens associated to HLA-I complex; 3) Inducing apoptosis in infected cells [6,7].

The enzyme-linked immunosorbent spot (ELISPOT) technique has been used in a crescent form for quantification of peripheral blood mononuclear cells (PBMC) producing specific cytokines in patients with infectious diseases $[2,8,9]$. There are several advantages of the ELISPOT assay for use in field settings: it is relatively easy to perform, does not require expensive instrumentation, and has the potential for high throughput screening. Through ELISPOT technique, several authors reported that the quantification of peripheral blood mononuclear cells producing IFN-gamma (PBMC-IG) presented cytotoxicity in response to influenzae peptide $[1,2,9]$. Several studies have shown that ELISPOT is more sensitive for detection of cytokines than ELISA technique [10-12]. However, there are not studies about PBMC-IG in patients with tuberculosis.

The discovery of the predominance of a specific cytokine in the inflammatory microenvironment, as well as in the peripheral blood, may help to understand the evolution of an infectious agent in the organism. The CD8+ T cells perform cytotoxic action mainly through major histocompatibility complex (MHC) class I molecules in association with CD1d molecules presenting glycolipids antigens to $\mathrm{T}$ lymphocyte cells [5].

In this study we quantified the PBMC-IG in patients with active tuberculosis employing the ELISPOT technique. Our results indicate that tuberculosis activates the Th1 immune response, and also that there is a positive correlation between PBMC-IG and some clinical and laboratory variables. 


\section{Material and Methods}

Patients Selection

Thirteen patients with active tuberculosis were selected from Infectious Diseases Clinic and Internal Medicine Clinic of the Clinics Hospital, School of Medicine, University of São Paulo. The control group had ten healthy individuals vaccinated against tuberculosis. Informed consent was obtained from each patient and the protocol for the research project was approved by the ethics committee of the institution.

We excluded patients with HIV test (ELISA) positive, and patients with immunodeficient diseases. Sera of patients and control group were obtained under sterile conditions and disposed in tubes containing sodium heparin $(0.1 \mathrm{mg} / \mathrm{mL})$. The maximum volume of the sample collected from each patient was $20 \mathrm{~mL}$. We collected blood samples of each patient in two moments: TBC1 = in admission without treatment; TBC2 = after seven to 20 days under treatment.

Peripheral Blood Mononuclear Cells Isolation

The PBMC isolation followed the protocol based in other studies [8,9] as follows: PBMC were isolated by centrifugation gradient with Ficoll-Paque Plus (Amersham). The cells were washed with saline solution and resuspended in Iscove's B medium (Sigma) with $4 \mathrm{mM}$ of l-glutamine (v/v), $100 \mathrm{U} / \mathrm{L}$ of penicillin, $100 \mathrm{U} / \mathrm{L}$ of streptomycin, enriched with $10 \%$ of human AB serum pool (Cultilab), and stored in fetal bovine serum containing DMSO 10\% in liquid nitrogen. The freezing technique was efficient, producing similar replies to fresh cells [13]. The isolated PBMC were incubated overnight in Iscove's medium containing fetal bovine serum (10\%) before ELISPOT assay.

The cells were counted in Neubauer chamber and disposed in a concentration of $1 \times 10^{4}$ cells $/ \mathrm{mL}$ in Iscove's medium, containing fetal bovine serum (10\%).

\section{ELISPOT Assay}

We added $100 \mathrm{~mL}$ of each sample (with $5 \times 10^{4}$ cells) of ten controls and 13 tuberculosis patients in a 96-well PVDF plate coated with a specific IFN-gamma antibody. The plate was incubated at $37^{\circ} \mathrm{C}$ in a $\mathrm{CO}_{2}$ incubator for $48 \mathrm{~h}$. The contents of the plate were discarded and the plate was thoroughly washed with $300 \mu \mathrm{L} /$ well of $1 \mathrm{x}$ wash buffer, taking care to not damage the membrane. This procedure was repeated for a total of six washes. After washing, the plate was turned upside down and blotted onto paper towels. We added $50 \mu \mathrm{L}$ of IFN-gammadetection antibody to each well. The plate was covered and incubated for $1 \mathrm{~h}$ at room temperature. After this, it was washed six times again. We added $100 \mu \mathrm{L}$ of diluted streptavidin-AP conjugate to each well. The plate was covered and incubated for one hour at room temperature, and it was washed six times again. Then, we added $100 \mu \mathrm{L}$ of NBT/BCIP substrate solution to each well. The color reaction was developed at room temperature, in the dark, for 3-15 minutes. The contents of the plate were discarded and it was rinsed with ultra pure water to stop the reaction. The plate was turned upside down, allowing the membrane to completely dry prior to analysis. The spotforming units (SFU) had been counted by using a dissection microscope (Quimis).

\section{Statistical Analysis}

The variables were tested about Gaussian distribution through Kolmogorov-Smirnov test (K-S) and Shapiro-Wilks test, and they were classified as parametric or nonparametric. The values were expressed as mean and standard deviation for parametric variables. In this group, we realized Student's t test unpaired for two groups comparison, and ANOVA test followed by Newman-Keuls post-test for comparison of more than two groups. Otherwise, the values were expressed as median and sample variability is reported as $25 \%$ and $75 \%$ percentiles for nonparametric variables. In this group we utilized the Kruskal-Wallis multiple comparisons test with Muller-Dunn post-test when comparing two or more unpaired groups and the unpaired Mann-Whitney test was used to compare two distinct groups.

The Pearson's correlation was made when indicated. An alpha risk equal or lower than $5 \%(\mathrm{p} \leq 0.05)$ was considered statistically significant.

\section{Results}

The data of patients' clinical status were: $9 / 13$ had weight loss ranging between three and $26 \mathrm{Kg}$; 8/13 had fever; 8/13 had cough without sputum; 6/13 had night sweats; 2/13 had cough with sputum. The diagnostic imaging tests showed: 10/13 had chest X-ray abnormal, of which 1/13 developed pulmonary cavitations, and 3/13 had signs of lymph nodes necrosis. The tuberculosis group had four women and nine men, and the control group had seven women and three men.

The tuberculosis patients had the following clinical forms: $9=$ pulmonary, $1=$ pleural, 1 = lymphatic + splenic, and $2=$ lymphatic. In relation to the clinical therapy, 10/13 did not have received anti-tuberculosis drugs at the time of blood sampling, and $3 / 13$ were using therapeutic scheme with isoniazid, rifampin and pyrazinamide for less than one week. The PPD skin test was positive in $4 / 13$. The C-reactive protein was high in $6 / 13$. The acid fast bacilli in sputum were positive in 11/13.

SFU counting of PBMC-IG of control group is shown in Table 1, and the SFU counting of tuberculosis group is shown in Table 2 (Figure 1). The numbers are concerning to spots number by $1 \times 10^{4}$ PBMC. The comparison of the spots counting between PBMC-IG and the control group are shown in Table 3. There was a significant difference of PBMC-IG counting between TBC1 (first sample) and control group ( $\mathrm{p}<$ 0.001 ) and between TBC2 (second sample) and control group $(\mathrm{p}<0.05)$, but there was no difference between TBC1 and TBC2 (p $>0.05)$.

The values (mean and standard deviation) of TBC1 and TBC2, age, weight loss, hemoglobin, erythrocyte sedimentation rate (ESR) and C-reactive protein (CRP) are shown in Table 4. A positive correlation was found between 
Table 1. Count of PBMC-IG spots of control group

\begin{tabular}{cc}
\hline Patients & Count of PBMC-IG \\
\hline 1 & 6 \\
2 & 2 \\
3 & 1 \\
4 & 1 \\
5 & 3 \\
6 & 2 \\
7 & 0 \\
8 & 2 \\
9 & 1 \\
10 & 0 \\
\hline
\end{tabular}

Table 2. Count of PBMC-IG of tuberculosis patients

\begin{tabular}{ccc}
\hline Patients & TBC1* & TBC2 \\
\hline 1 & 5 & 0 \\
2 & 26 & 10 \\
3 & 23 & 9 \\
4 & 16 & 21 \\
5 & 27 & 25 \\
6 & 21 & 18 \\
7 & 3 & - \\
8 & 23 & - \\
9 & 30 & 24 \\
10 & 45 & - \\
11 & 11 & 12 \\
12 & 12 & 21 \\
13 & 65 & 13 \\
\hline
\end{tabular}

Note: $*=$ first sample; $* *=$ second sample

Table 3. Comparison of PBMC-IG counts among TBC1, TBC2 and control group in patients with tuberculosis

\begin{tabular}{lccc}
\hline & Control & TBC1 & TBC2 \\
\hline Mean & 1.80 & $23.62^{* *}$ & $15.30^{* *}$ \\
Standard-Deviation & 1.75 & 16.73 & 7.89 \\
Standard-Error & 0.55 & 4.64 & 2.50 \\
\hline
\end{tabular}

Note: We used the ANOVA one-way test. ${ }^{* *} \mathrm{p}=0.0004$. Statistical difference among groups (Newman-Keuls post-test): Control versus TBC1 $=\mathrm{p}<0.001$; Control versus TBC2 $=\mathrm{p}<0.05$; TBC2 versus $\mathrm{TBC} 1=\mathrm{p}>0.05$ (not significant).

Table 4. Values of mean and standard-deviation of PBMC-IG counts for TBC1, TBC2, clinical and laboratory variables in patients with tuberculosis

\begin{tabular}{lccc}
\hline & Patients (N) & Mean & Standard-deviation \\
\hline TBC1 & 13 & 23.62 & 16.731 \\
TBC2 & 10 & 15.30 & 7.889 \\
Age & 13 & 40.54 & 16.184 \\
Weight loss & 8 & 8.13 & 7.936 \\
Hemoglobin & 12 & 12.833 & 1.8763 \\
ESR & 11 & 46.64 & 36.694 \\
CRP & 7 & 78.537 & 65.4619 \\
\hline
\end{tabular}

Figure 1. Comparison of spots obtained in control and TBC patient groups.
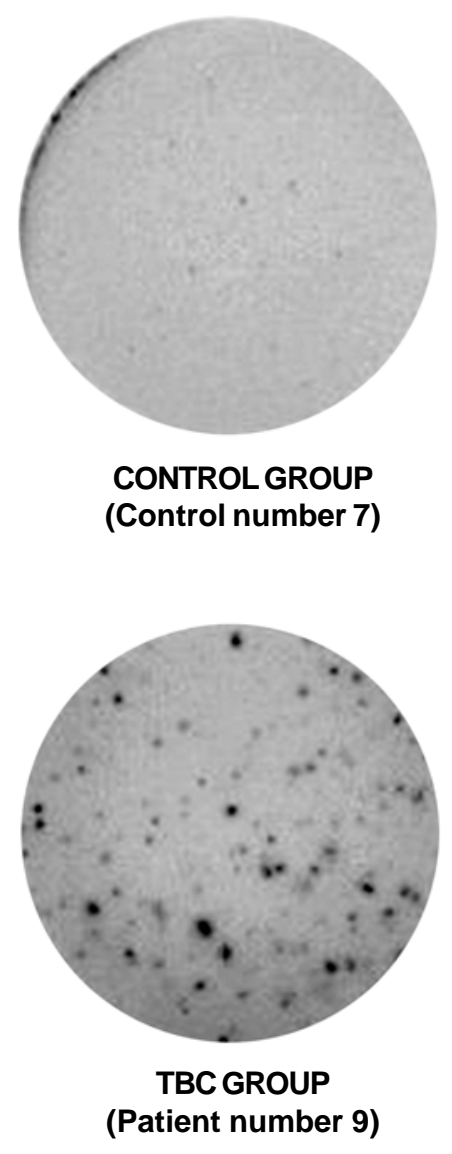

PBMC-IG and hemoglobin value (Pearson correlation = 0.714; $\mathrm{p}=0.031 ; \mathrm{n}=9$ ), as well as between PBMC-IG and weight loss (Pearson correlation $=0.702 ; \mathrm{p}=0.05 ; \mathrm{n}=8$ ) degree. There was no correlation between PBMC-IG and other variables.

\section{Discussion}

The results showed that ELISPOT method used here was efficient for measurement of PBMC-IG in patients with active tuberculosis. We verified that patients with active tuberculosis produce significantly higher PBMC-IG in relation to control group. These results suggest that tuberculosis activates Th1 immune response due to increase of PBMC-IG. IFN-gamma is a product mainly of natural killer cells (NK), activates T cells, and has an important role in host defense against mycobacteria. IFN-gamma modulates the macrophages activation, increases MHC class II molecules expression and antigen presentation, and also increases differentiation of lymphocyte populations [14]. This cytokine has been measured ex vivo in serum [15], bronchoalveolar lavage fluids [16] and pleural effusions of TBC patients [17]. Out of seven studies using ELISPOT, for found that TBC patients produce more IFN-gamma than controls [18-21], one found that they produce less [22], and two found no difference [23,24]. 
Therefore, in order to establish definitive conclusions about these discrepant results, more studies should be done.

In patients with active tuberculosis, the PBMC-IG count in second sample (TBC2) was lesser than PBMC-IG count in first sample (TBC1), but without statistical difference. TBC2 determination was made within seven to 20 days of the beginning of anti-tuberculosis treatment. In the same way, Bekker et al. [25] showed that the serum IFN-gamma levels decreased in patients with TBC after beginning of therapeutic. Hirsch et al. [26] reported that IFN-gamma production was lower at the TBC diagnosis, and this phenomenon remains for 12 months. The authors suggested that TBC can induce a lesser response of $\mathrm{T}$ lymphocytes during and after infection. We speculate that the stability of PBMC-IG values between TBC1 and TBC2 can have occurred due to treatment influence, and to the consequent decrease of the bacterial load in each case. Another possibility is that the immune response reaches a plateau in normal conditions, or it suffers inhibition for still unknown mechanism.

In the analysis of the correlation between PBMC-IG levels and clinical and laboratory variables, we found a positive correlation among PBMC-IG and hemoglobin and weight loss. This result indicates that may be a link between patient's clinical status and the immune response intensity. We may consider that greater weight loss indicates higher bacterial load. In relation to hemoglobin level, it is possible that a patient with higher hemoglobin is in better clinical condition, and its immune response will be better too. The correlations between PBMCIG and clinical and laboratory variables may indicate that the immune response against tuberculosis are not "neck or nothing” type. It is possible that the immune response may be modulated by other factors, as patient's clinical status. We did not find a correlation between PBMC-IG and C-reactive protein, as well as between PBMC-IG and erythrocyte sedimentation rate. These two clinical tests may not have correlation with the true inflammatory response that is occurring.

The positive correlation between weight loss and PBMCIG is coherent with other studies that had found correlation between serum TNF-alpha levels and weight loss. TNF-alpha and IFN-gamma take part in the Th1 immune response. Bossola et al. [27] showed that serum TNF-alpha concentrations were significantly higher in patients with cancer and severe weight loss when compared with patients having cancer and low weight loss. Cakir et al. [28] stated that serum TNF-alpha level and leptin might be responsible for the weight loss in pulmonary tuberculosis patients. Another study also correlated chronic administration of TNF-alpha with weight loss in rats [29].

Another existing knowledge indicates that both TNFalpha and IFN-gamma participate in the granuloma formation and they also appear crucial to the formation of $M$. tuberculosis-constraining granuloma. Beyond this effect, IFNgamma seems to exert a main role as endogen activator agent for murine macrophages and their antimycobacterial effects
[30,31]. Other study showed that IFN-gamma actuates synergistically with TNF-alpha for these antimycobacterial effects [32].

Our study suggests that true immunological tests as ELISPOT for PBMC-IG detection must be added to therapeutical arsenal for patients with TBC. The greater knowledge about immune response type in infectious diseases provides new perspectives for treatment of these diseases in a near future. The predominance of Th1 immune response induces a greater cellular death by necrosis or apoptosis, reaching both host and inflammatory cells. We reasoned that the inhibition of Th1 response for reduction of tissue injury might be considered in selected cases. We think that the modulation of the immune response besides of the treatment of infectious agents will be part of infectious diseases therapy.

\section{References}

1. Lalvani A., Brookes R., Wilkinson R.J., et al. Human cytolytic and interferon-gamma secreting CD8+ T lymphocytes specific for Mycobacterium tuberculosis. Proc Natl Acad Sci USA 1998;95:270-5.

2. Scheinbenbogen C., Lee K.H., Stevanovic S., et al. Analysis of T cell response to tumor and viral peptide antigens by an IFNgamma- "Elispot” assay. Int J Cancer 1997;71:932-6.

3. Shellito J.E., Tate C., Ruan S., Kolls J. Murine CD4+ T lymphocyte subsets and host defense against Pneumocystis carinii. J Infect Dis 2000;181:2011-7.

4. Tilley P.A.G., Menon J.N. Detection of Mycobacterium specific interferon-gamma producing human $\mathrm{T}$ lymphocytes by flow cytometry. APMIS 2000;108:57-66.

5. Park S.H., Bendelac A. CD1-restricted T-cell responses and microbial infection. Nature 2000; 406:788-92.

6. Lewinsohn D.M., Briden A.L., Reed S.G., et al. Mycobacterium tuberculosis reactive CD8+ T lymphocytes: the relative contribution of classical versus nonclassical HLA restriction. J Immunol 2000; 165: 925-30.

7. Serbina N.V., Liu C.C., Scanga C.A., et al. CD8+ CTL from lungs of Mycobacterium tuberculosis infected mice express perforin in vivo and lyse infected macrophages. J Immunol 2000; 165: 353-63.

8. Asemissen A.M., Nagorsen D., Keilholz U., et al. Flow cytometric determination of intracellular or secreted IFN-gamma for the quantification of antigen reactive $\mathrm{T}$ cells. J Immunol Methods 2001;251:101-8.

9. Schimittel A., Keilholz U., Scheinbenbogen C. Evaluation of the interferon-gamma-Elispot-assay for quantification of peptide specific $\mathrm{T}$ lymphocytes from peripheral blood. J Immunol Methods 1997; 210:167-74.

10. Ekerfelt C., Ernerudh J., Jenmalm M.C. Detection of spontaneous and antigen-induced human interleukin-4 responses in vitro: comparison of ELISPOT, a novel ELISA and real-time RTPCR. J Immunol Methods 2002;260:55-67.

11. Makitalo B., Andersson M., Arestrom I. ELISpot and ELISA analysis of spontaneous, mitogen-induced and antigen-specific cytokine production in cynomolgus and rhesus macaques. J Immunol Methods 2002; 270:85-97.

12. Tanguay S., Killion J.J. Direct comparison of ELISPOT and ELISAbased assays for detection of individual cytokine-secreting cells. Lymphokine Cytokine Res 1994;13:259-63.

13. McCutcheon M., Wehner N., Wensky A., et al. A sensitive ELISPOT assay to detect low-frequency human $\mathrm{T}$ lymphocytes. J Immunol Methods 1997;210:149-66.

14. Casanova J.L., Abel L. Genetic dissection of immunity to mycobacteria: the human model. Annu Rev Immunol 2002;20: 581-620. 
15. Vankayalapati R., Wizel B., Weis S.E., et al. Serum cytokine concentrations do not parallel Mycobacterium tuberculosisinduced cytokine production in patients with tuberculosis, Clin Infect Dis 2003;36:24-8.

16. Barry S.M., Lipman M.C., Bannister B., et al. Purified protein derivative-activated Type 1 cytokine-producing CD4+ T lymphocytes in the lung: a characteristic feature of active pulmonary and nonpulmonary tuberculosis. J Infect Dis 2003;187: 243-50.

17. Hirsch C.S., Toossi Z., Johnson J.L., et al. Augmentation of apoptosis and interferon- $\gamma$ production at sites of active Mycobacterium tuberculosis infection in human tuberculosis. J Infect Dis 2001;183:779-88.

18. Ulrichs T., Anding P., Porcelli S., et al. Increased numbers of ESAT-6- and purified protein derivativespecific gamma interferon-producing cells in subclinical and active tuberculosis infection. Infect Immun 2000;68: 6073-6.

19. Lalvani A., Pathan A.A., McShane H., et al. Rapid detection of Mycobacterium tuberculosis infection by enumeration of antigenspecific T cells. Am J Respir Crit Care Med 2001;163: 824-8.

20. Ferrand R.A., Bothamley G.H., Whelan A., et al. Interferongamma responses to ESAT-6 in tuberculosis patients early into and after anti-tuberculosis treatment. Int $\mathrm{J}$ Tuberc Lung Dis 2005;9:1034-9.

21. Morosini M., Meloni F., Uccelli M., et al. Ex vivo evaluation of PPD-specific IFN- $\gamma$ or IL-5 secreting cells in the peripheral blood and lungs of patients with tuberculosis. Int J Tuberc Lung Dis 2005;9:753-9.

22. Shams H., Wizel B., Weis S.E., et al. Contribution of CD8+ T cells to gamma interferon production in human tuberculosis. Infect Immun 2001;69:3497-501.

23. Surcel H.M., Troye-Blomberg M., Paulie S., et al. Th1/Th2 profiles in tuberculosis, based on the proliferation and cytokine response of blood lymphocytes to mycobacterial antigens. Immunology 1994; 81: 171-6.
24. Vincenti D., Carrara S., De Mori P., et al. Identification of early secretory antigen target-6 epitopes for the immunodiagnosis of active tuberculosis, Mol Med 2003;9:105-11.

25. Bekker L.G., Maartens G., Steyn L., Kaplan G. Selective increase in plasma tumor necrosis factor alpha and concomitant clinical deterioration after initiating therapy in patients with severe Tuberculosis. J Infect Dis 1998;178:580-4.

26. Hirsch C.S., Toossi Z., Othieno C. et al. Depressed T-cell interferongamma responses in pulmonary tuberculosis: analysis of underlying mechanisms and modulation with therapy. J Infect Dis 1999;180:2069-73.

27. Bossola M., Muscaritoli M., Bellantone R., et al. Serum tumour necrosis factor - alpha levels in cancer patients are discontinuous and correlate with weight loss. Eur J Clin Invest 2000; 30:1107-12.

28. Cakir B., Yonem A., Guler S., et al. Relation f leptin and tumor necrosis factor alpha to body weight changes in patients with pulmonary tuberculosis. Horm Res 1999; $52: 279-83$.

29. Ling P.R., Schwartz J.H., Bristrian B.R. Mechanisms of host wasting induced by administration of cytokines in rats. Am J Physiol 1997; 272:E333 - E9.

30. Rook G.A.W., Steele J., Ainsworth M., Champion B.R. Activation of macrophages to inhibit proliferation of Mycobacterium tuberculosis: comparison of the effects of recombinant gamma interferon on human monocytes and murine peritoneal macrophages. Immunol 1986;59:333-8.

31. Flesch I., Kaufmann S. Mycobacterial growth inhibition by interferon-g activated bone marrow macrophages and differential susceptibility among strains of Mycobacterium tuberculosis. J Immunol 1987;138:4408-13.

32. Flesch I., Kaufmann S. Activation of tuberculostatic macrophage functions by gamma interferon, interleukin4 , and tumor necrosis factor. Infect Immun 1990;58:2675-7. 